CLINICAL STUDY

\title{
Morbidity and GH deficiency: a nationwide study
}

Kirstine Stochholm, Torben Laursen ${ }^{1}$, Anders Green ${ }^{2,3}$, Peter Laurberg ${ }^{4}$, Marianne Andersen ${ }^{5}$, Lars Østergaard Kristensen ${ }^{6}$, Ulla Feldt-Rasmussen ${ }^{7}$, Jens Sandahl Christiansen, Morten Frydenberg ${ }^{8}$ and Claus Højbjerg Gravholt

Medical Department M (Endocrinology and Diabetes), Aarhus University Hospital, Aarhus Sygehus, NBG, DK-800O Aarhus C, Denmark, ${ }^{1}$ Department of Pharmacology, University of Aarhus, 8000 Aarhus C, Denmark, ${ }^{2}$ Department of Applied Research and HTA, Odense University Hospital, 5000 Odense C, Denmark, ${ }^{3}$ Institute of Public Health, University of Southern Denmark, 5000 Odense C, Denmark, ${ }^{4}$ Department of Endocrinology, Aalborg University Hospital, 9100 Aalborg, Denmark, ${ }^{5}$ Department of Endocrinology, Odense University Hospital, 5000 Odense C, Denmark, ${ }^{6}$ Department of Endocrinology, Herlev University Hospital, 2730 Herlev, Denmark, ${ }^{7}$ Department of Endocrinology PE, Rigshospitalet, 2100 Copenhagen, Denmark and ${ }^{8}$ Department of Biostatistics, University of Aarhus, 8000 Aarhus C, Denmark

(Correspondence should be addressed to K Stochholm; Email: stochholm@dadlnet.dk)

\begin{abstract}
Objective: To estimate morbidity in Denmark in all patients with GH deficiency (GHD).

Design: Morbidity was analyzed in 1794 GHD patients and 8014 controls matched on age and gender. All records in the GHD patients were studied and additional morbidity noted. Diagnoses and dates of admissions were identified in the National Patient Registry. Lag time until first admission was used as a measure of morbidity. Patients were divided into childhood onset (CO) and adult onset (AO), discriminated by an age cut-off of 18 years at onset of GHD.

Method: Sex- and cause-specific hazard ratios (HRs) in CO and AO GHD compared with controls.

Results: Total morbidity was significantly increased in the GHD patients. HR for CO males: $3.1(95 \%$ confidence interval (CI): 2.7-3.7), CO females: 3.2 (95\% CI: 2.6-3.9), AO males: 2.9 (95\% CI: 2.6-3.2), and AO females: 3.2 (95\% CI: 2.8-3.6). In 18 out of 20 chapters from the International Classification of Diseases-10, a significantly increased morbidity was identified for at least one of the four subgroups of patients. Morbidity was significantly increased in all the four subgroups due to infectious, endocrine, pulmonary, urogenital, and neurological diseases; cancer; diseases of the eye, ear, and circulatory diseases; and traumas. Fractures were significantly increased in AO females, not in males.

Conclusions: Morbidity was significantly increased in the GHD patients. The increased morbidity was due to a variety of disorders, some of which can readily be explained by GHD and other pituitary deficiencies, while others cannot be easily explained.
\end{abstract}

European Journal of Endocrinology 158 447-457

\section{Introduction}

The morbidity in patients with growth hormone deficiency (GHD), often in addition to other pituitary deficiencies, has been described in a number of clinical papers, typically focusing on clinical measures of disease, for instance cardiovascular risk markers. In GHD children, there is an increased risk of fractures (1), and reduced bone mineral density (BMD) has been reported (2). Furthermore, the treatment (e.g., irradiation) of children with pituitary disease eventually aggravates these disturbances, and may to some extent eclipse the relative impact of GHD (3). A decreased severity of cardiovascular risk factors in adults with childhood onset (CO) compared with adult onset (AO) craniopharyngeoma was identified (4). In adult GHD patients, the metabolic changes after treatment with GH have been described in detail $(5,6)$. Finally, several studies have shown that GH replacement improves cardiovascular risk factors (7), tends to normalize body composition (8), and induces anabolic changes (9). A variety of disorders such as cancer, gene defects, apoplexies, trauma, and others might result in GHD in children or adults. Hypopituitary patients comprise a heterogeneous group, and an increased morbidity in GHD patients is a natural consequence of these clinical findings. However, morbidity in hypopituitary adults, including GHD, has only been described in one epidemiological study (10). Here, morbidity in GH-treated adults with GHD and in a relatively older group of non-GH-treated hypopituitary patients was studied. No difference in cancer or cerebrovascular events in $289 \mathrm{GHD}$ patients on GH treatment compared with the background population was identified. By contrast, these events were significantly increased in the non-GH-treated hypopituitary patients. Among children and adolescents treated with human pituitary GH, the risk of secondary neoplasia during 1959-1985 was increased (11).

In this nationwide study of patients suffering from GHD, a diagnosis verified by a thorough examination of 
individual records, the total and cause-specific morbidities compared with age- and gender-matched controls are described.

\section{Materials and methods}

The patients have previously been described in detail concerning identification in registries and definition of the GHD diagnosis (12) and concerning mortality (13). Three registries identified the primary cohort: the Cancer Registry (CR) that registers patients in Danish hospitals with a cancer diagnosis; the National Patients Registry (NPR) that registers all inpatients and, since 1995, all outpatients; and the Causes of Death Registry (CDR) that registers causes of death in all deceased Danes.

In CR, we applied for topography in proximity to the pituitary gland and both benign and malignant morphologies. In NPR, we searched for all hospital activities coded with diagnoses with an increased risk of GHD, using International Classification of Diseases 8th edition (ICD-8) and ICD-10 (12, 13). From CDR, we identified all cases with specific hypopituitary diagnoses. The cases from CR, NPR, and CDR all had an increased a priori risk of having GHD.

Criteria for the GHD diagnosis were based on clinical and laboratory findings. The interpretation of the stimulation tests were based on current consensus guidelines $(14,15)$. The criteria for patients without stimulation tests were based on data from studies in patients with previous radiotherapy (16), with two or more additional pituitary hormone deficiencies (17), and with insulin-like growth factor-I (IGF-I) below -2 s.D. (15), showing that such patients with high probability also suffer from GHD. It is important to stress that the diagnosis GHD was only applied when the clinical context was relevant, in order to minimize the inclusion of false-positive persons. In short, we identified 9131 possible GHD cases in three national registries and verified the diagnosis of GHD by studying their individual records, during which we visited 125 hospitals and archives. All departments with registrations gave permission to read the records. In total, 2205 patients fulfilled our criteria of GHD.

In describing the cohort, the following dates are important: 1) the date of onset represents the estimated date the patient became GH deficient; 2) the date of sufficient information represents the date when the date of onset was notified in a record; and 3) the date of registration represents the first date when the patient is noted in any register. For each patient, the date of entry of GHD was defined as 1) the date of first registration in the registry or 2) the date of sufficient information that ever came last. It is necessary to use the latest date, since patients must have been both registered to enter the primary cohort of possible cases, and the record must contain sufficient information to ensure a correct diagnosis of GHD. The entry date is considered as the incident date and does not necessarily represent a diagnosis at the hospital, since only some of the patients had been formally tested for GHD at any department. The date of onset is not used for the calculation of start of risk time, and rather used to give a rough estimate of the difference between the dates of entry and onset, defined as 'delay'. Delay represents the period during which patients had GHD without contributing to followup time. The date of entry occurred during 1980-1999 in 1823 patients.

The patients divided into adult and CO GHD were discriminated by a cut-off age of 18 years at onset. Patients with AO were divided into four age groups according to age at the date of entry: $<45$ years of age, between 45 and 54 years, between 55 and 64 years, and 65 years of age or more.

To obtain controls, we used The Central Office of Civil Registration (OCR). Here, vital statistical information including birth, death, and emigration for Danish citizens is centralized using the unique central person number. On August 6, 2004, OCR matched the 1823 patients with up to five controls each. The controls were matched on gender and age (month and year of birth) and were alive on the date of entry. For 29 patients, no controls were identified; they were omitted from all calculations. We retrieved 8014 controls for 1794 patients. These patients and controls were used for all calculations, unless specified differently.

Since 1977, the National Patient Registry (NPR) has been registering all diagnoses at discharge of inpatients, and from 1995 all in- and outpatients linked to the date of admission. The registration is compulsory and ICD-8 was used during 1977-1993, and ICD-10 during 1994 to the present. Diagnoses were translated to ICD-10. The diagnoses and the corresponding date of admission for patients and controls were identified in NPR September 10, 2005. All admissions without discharge before this date were not included in the identification. However, as follow-up ended on August 6, 2004, the number of admissions not identified is limited. Every admission had one primary diagnosis and a number (including none) of secondary diagnoses. For all calculations, diagnoses that led to the initial identification of the 9131 possible GHD patients (12) were omitted. Other diagnoses registered at the same admission as these particular diagnoses were included, unless specified differently. Morbidity was defined as lag time from the date of entry until first relevant diagnosis in NPR comparing patients and controls. In case of no diagnoses, end of follow-up was either the date of death or emigration, or August 6 , 2004 , whichever came first. For the calculation of causespecific morbidity, we divided all diagnoses into 20 ICD10 chapters. For each chapter, the first diagnosis was identified and used for the calculation of lag time. Similarly, for total morbidity the very first diagnosis after entry was used, regardless of the chapter.

Based on the information from clinical studies of the consequences of hypopituitarism, the following 
chapters were selected and divided into sub-chapters according to ICD-10: endocrine diseases, circulatory diseases, and pregnancy and childbirth. Furthermore, morbidity due to fractures was identified and analyzed. The sub-chapter analyses were performed on males and females with AO only. We chose not to analyze the cancer chapter in more detail, since it had been planned to use a more stringent approach employing registrations from the CR in a future analysis.

The patients have per se an increased risk of admittance to a hospital compared with the controls and hence an increased risk of being diagnosed with a disease that in itself would not lead to an admission (18). To evaluate the possible effect of this bias, we repeated the analysis applying two other approaches: 1) omitting all admissions with diagnoses used for the initial identification of the patients and 2) only considering primary diagnoses. Furthermore, as death can be considered the ultimate morbidity, deaths (excluding accidents) occurring without a diagnosis at a hospital will underestimate morbidity in both controls and patients, but not necessarily induce bias in the comparison. We repeated the analysis on total morbidity including death as admittance to a hospital.

Deficiencies of the other pituitary axes were defined as follows: adrenocorticotrophin: an insufficient response to metyrapone or synacthen, thyrotrophin: blood sample combined with a clinical decision of deficiency, follicle-stimulating hormone/luteinizing hormone: blood sample combined with a clinical decision of deficiency or a clinical definition of deficiency, and antidiuretic hormone: a positive thirst test or clinical suspicion of deficiency combined with successful medical treatment for more than 3 months. The patients were defined as deficient on the relevant axis if one of the above was deficient at any stage, or they were substituted for the axis.

The study was approved by The Danish Data Protection Agency, The Research Ethics Committee, and Doctors' Counsel in The Danish National Board of Health.

\section{Statistical analysis}

The characteristics of the patients with CO compared with AO GHD were compared using the $\chi^{2}$ test. Morbidity was defined as lag time from the date of entry until admission with a relevant diagnosis in NPR. Morbidity was analyzed with Cox regression using each patient and the matched controls as a stratum. Hereby, the comparisons were adjusted for gender, age, and calendar time. Differences in relative morbidity with age at entry were analyzed using Cox regression applying age at entry as a linear variable. For the analysis of morbidity within the patients, we used Cox regression stratified on age at entry (5-year intervals); no controls were included in this analysis. There was no correction made for multiple comparisons. All results are shown with 95\% confidence limits and $P<0.05$ was considered statistically significant. Stata 8.2 (Stata Corp., College Station, TX, USA) was used for all calculations.

\section{Results}

The characteristics of the four subgroups are described in Table 1. Up to $30 \%$ of patients had been irradiated, most being $\mathrm{CO}$, while $\sim 70 \%$ of $\mathrm{AO}$ were subjected to surgery.

For the following analyses, it is important to note that morbidity data are compared with controls, unless specified differently.

\section{Total morbidity}

We identified a significantly increased total morbidity in the GHD patients and no difference in morbidity between the genders in the four subgroups (Table 2). Analyzing patients only, no gender-specific difference in total morbidity was identified in CO (Hazard ratio (HR): 1.0, 95\% confidence interval (CI): 0.8-1.2); however, females with AO GHD had a significantly increased morbidity compared with males (HR: 1.1, 95\% CI: 1.0-1.3).

Death was included as admittance to a hospital, and the significantly increased total morbidity changed only marginally for the four subgroups (data not shown).

\section{Cause-specific morbidity}

HR was calculated in 20 chapters in both genders in $\mathrm{CO}$ (Fig. 1) and AO GHD (Fig. 2; Table 3). For all the four subgroups, there was a significantly increased morbidity due to infectious, cancer, endocrine, neurological, circulatory, pulmonary, and urogenital diseases; diseases of the eye and ear; trauma; and observations (corresponding to admissions where a disease had been suspected, but not identified). Females with childhood and AO GHD had significantly decreased HRs when calculating the sub-chapter of pregnancy and childbirth.

We then analyzed data 1) excluding all admissions with diagnoses used for primary identification of the patients and 2) including only primary diagnoses (Table 3); generally, however, most HRs were still significant.

\section{Cause-specific morbidity (sub-chapters)}

In endocrine and circulatory diseases, we identified several sub-chapters with significantly increased morbidity for AO GHD patients, whereas the morbidity due to pregnancies was significantly decreased in two subchapters (Table 4). Furthermore, we pooled all fracture diagnoses and identified the HR for males 1.0 (95\% CI: $0.8-1.3$ ) and females 1.4 (95\% CI: 1.1-1.7). 
Table 1 The characteristics of patients with growth hormone deficiency (GHD) in childhood onset (CO) and adult onset (AO) discriminated by an age cut-off below or above 18 years at onset of GHD. Onset is when the patients became GHD, entry is when follow-up begins, and delay is the difference between entry and onset.

\begin{tabular}{|c|c|c|c|c|}
\hline & \multicolumn{2}{|c|}{ CO } & \multicolumn{2}{|c|}{ AO } \\
\hline & Males & Females & Males & Females \\
\hline Number of patients ${ }^{a}$ & $299(9)$ & $187(7)$ & $732(40)$ & $576(28)$ \\
\hline Number of controls ${ }^{\mathrm{a}}$ & $1332(187)$ & $872(131)$ & $3200(417)$ & $2610(284)$ \\
\hline $\begin{array}{l}\text { Median lag time (range) until first diagnosis } \\
\text { (years) patients }\end{array}$ & $1.0(0.0-20.4)$ & $0.8(0.0-21.7)$ & $0.8(0.0-22.3)$ & $0.6(0.0-18.8)$ \\
\hline $\begin{array}{l}\text { Median lag time (range) until first diagnosis } \\
\text { (years) controls }\end{array}$ & $4.9(0.0-24.0)$ & $5.4(0.0-23.3)$ & $4.1(0.0-24.3)$ & $3.7(0.0-24.4)$ \\
\hline Median age (range) at onset (years) & $8.9(0.0-18.0)$ & $8.3(0.0-18.0)$ & $52.4(18.1-91.3)$ & $48.8(18.0-87.6)$ \\
\hline Median age (range) at entry (years) & $13.5(0.1-77.0)$ & $12.9(0.1-78.8)$ & $57.2(18.1-91.3)$ & $55.8(18.5-87.7)$ \\
\hline Median delay (range) (years) & $1.0(0.0-61.0)$ & $1.2(0.0-62.4)$ & $0.2(0.0-48.8)$ & $0.3(0.0-56.2)$ \\
\hline Percentage diagnosed $\mathrm{GHD}^{\star, \mathrm{b}}$ & 73.3 & 73.8 & 30.0 & 29.7 \\
\hline Irradiation $(\%)^{*}$ & 30.8 & 28.3 & 19.8 & 21.0 \\
\hline Surgery $(\%)^{*}$ & 32.1 & 37.4 & 70.9 & 67.7 \\
\hline No other deficits $(\%)^{\star}$ & 34.1 & 35.3 & 6.3 & 9.2 \\
\hline 1-2 additional deficits (\%) & 36.8 & 32.1 & 33.2 & 46.5 \\
\hline 3-4 additional deficits $(\%)^{\star}$ & 29.1 & 32.6 & 60.5 & 44.3 \\
\hline Treatment with chemotherapy $(\%)^{*}$ & 10.4 & 7.5 & 1.8 & 1.6 \\
\hline Substitution on GH axis $(\%)^{*}$ & 77.3 & 74.9 & 24.9 & 22.2 \\
\hline \multicolumn{5}{|l|}{ Number of patients deficient (substituted) } \\
\hline $\mathrm{ADH}$ axis & $54(53)$ & $35(32)$ & $110(101)$ & $113(104)$ \\
\hline TSH axis & $135(128)$ & $87(87)$ & $538(506)$ & 395 (370) \\
\hline ACTH axis & 115 (109) & 79 (73) & 581 (559) & $437(425)$ \\
\hline $\mathrm{FSH} / \mathrm{LH}$ axis $^{\mathrm{c}}$ & $147(121)$ & $91(75)$ & $268(241)$ & $198(150)$ \\
\hline
\end{tabular}

$\mathrm{ADH}$, antidiuretic hormone; TSH, thyroid stimulating hormone; $\mathrm{ACTH}$, adrenocorticotrophin; $\mathrm{FSH}$, follicle-stimulating hormone; $\mathrm{LH}$, luteinizing hormone.

${ }^{*} \mathrm{CO}$ versus $\mathrm{AO} P<0.05$

${ }^{\mathrm{a}}$ Numbers in parentheses are those with no diagnoses. Diagnoses used for the identification of patients are excluded.

${ }^{\mathrm{b}}$ The percentage of patients with a clinical diagnosis of GHD.

${ }^{\mathrm{c}}$ For $\mathrm{AO} \mathrm{GHD}$, only patients $<55$ years at entry.

\section{Age-specific morbidity}

In the age groups 45-54 and 55-64 years, we identified an increased total morbidity in females compared with males; however, this was significant only in the latter age group $(P<0.05$; Table 2$)$. We found a significant reduction in total morbidity with advancing age at entry in males $(P<0.05)$, but not in females $(P=0.58)$. This reduction in morbidity with advancing age at entry was also present in the following chapters for males: cancer; endocrine, mental, neurological, eye, ear, pulmonary, gastrointestinal and urogenital diseases; and trauma $(P<0.05$ for all; Table 5$)$.

\section{Subgroup analysis}

Cause-specific morbidity was calculated for three different subgroups: excluding all patients with a malignant or possibly malignant cause of GHD and craniopharyngeoma, excluding all patients with former acromegaly or Cushing's syndrome, and excluding all patients who received irradiation as well as their controls. All calculations were repeated corresponding to Table 3 (data not shown). Most significant HRs remained significantly elevated (Table 3). After the exclusion of patients who received irradiation, the HR for the cancer chapter was still increased (males' CO:

Table 2 Hazard ratios (HRs) of total morbidity in childhood onset (CO) and adult onset (AO) growth hormone deficiency (GHD). Furthermore, morbidity in female versus male patients, both compared with controls, and number of patients is given.

\begin{tabular}{lcccccc}
\hline & Co & \multicolumn{5}{c}{ AO } \\
\cline { 3 - 7 } & \multicolumn{1}{c}{ Total } & Total & $<45$ & $45-54$ & $55-64$ & $65+$ \\
\hline Males & $3.1(2.7-3.7)$ & $2.9(2.6-3.2)$ & $3.5(2.8-4.3)$ & $2.8(2.2-3.5)$ & $2.9(2.4-3.6)$ & $2.4(2.0-2.9)$ \\
Females $_{\text {Number of patients }}{ }^{\mathrm{a}}$ & $3.2(2.6-3.9)$ & $3.2(2.8-3.6)$ & $2.8(2.3-3.5)$ & $3.8(2.9-5.1)$ & $4.2(3.2-5.6)$ & $2.7(2.2-3.4)$ \\
Number of controls $^{\mathrm{a}}$ & $486(299)$ & $1308(732)$ & $359(181)$ & $247(146)$ & $303(185)$ & $399(220)$ \\
Females versus males $^{204(1332)}$ & $5810(3200)$ & $1695(851)$ & $1151(678)$ & $1341(803)$ & $1623(868)$ \\
& $1.0(0.8-1.3)$ & $1.1(0.9-1.3)$ & $0.8(0.6-1.1)$ & $1.4(1.0-2.0)$ & $\mathbf{1 . 4 ( 1 . 0 - 2 . 1 )}$ & $1.1(0.8-1.5)$ \\
\hline
\end{tabular}

$\mathrm{CO}$ and $\mathrm{AO}$ were discriminated by an age cut-off below or above 18 years at onset of GHD. AO was subdivided according to age at entry. Significant $\mathrm{HRs}$ are given in bold.

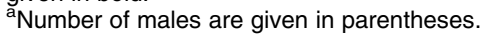




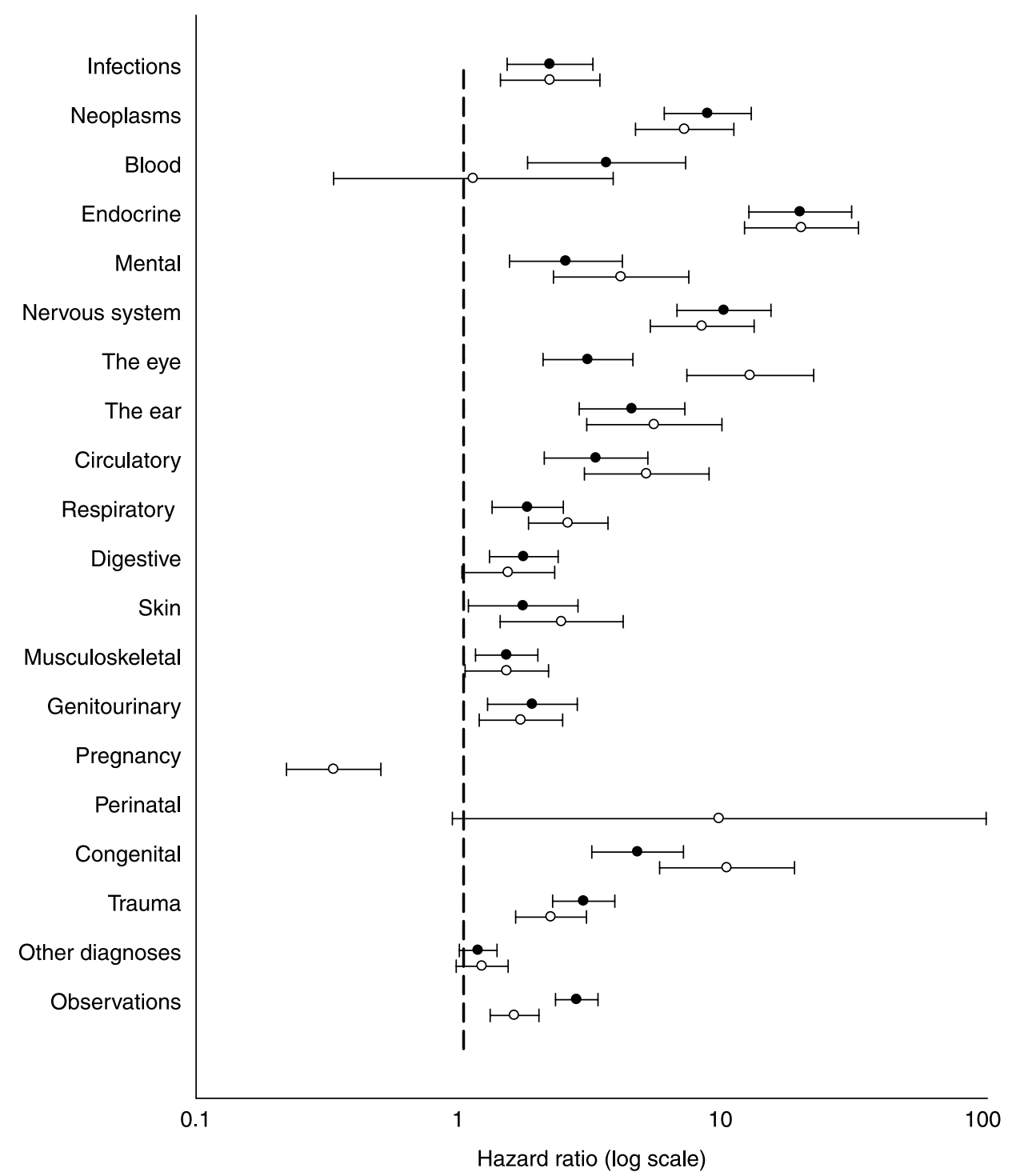

Figure 1 Hazard ratios of morbidity in childhood onset (CO) GH deficiency (GHD) divided into 20 ICD-10 chapters. CO patients have an age at onset of GHD of $<18$ years. Males are represented in black circles and females in white circles.

5.4 (95\% CI: 3.4-8.5), females' CO: 4.6 (95\% CI: 2.7-7.8), males' AO: 2.0 (95\% CI: 1.6-2.4), and females' AO: 1.7 (95\% CI: 1.3-2.0)). Furthermore, we calculated total morbidity for the four subgroups in patients with isolated GHD, in patients who had been operated, in patients who had been irradiated, and who were treated with GH (Table 6).

\section{Discussion}

In this first nationwide study on morbidity in patients with GHD, we identified a significantly increased total morbidity in childhood and AO GHD for both genders compared with controls. In 18 out of 20 ICD-10 chapters, at least one of the four subgroups had a significantly increased morbidity. Furthermore, morbidity significantly decreased with advancing age at entry in AO males.

The major findings were the significantly increased total morbidity in all the four subgroups of GHD patients, even after exclusion of high-risk patients. However, these data do not identify whether the increased morbidity is due to the pituitary status, treatment or lack of treatment hereof, surgery, or irradiation, but rather that the morbidity in the GHD patients as a cohort is significantly increased and thus probably reflects the consequences of pituitary disease. In patients with $\mathrm{AO} \mathrm{GHD}$, about the same number of males and females received treatment with $\mathrm{GH}$; thus we believe that $\mathrm{GH}$ treatment cannot explain the increased morbidity in female versus male patients.

We analyzed morbidity due to endocrine diseases in sub-chapters. We identified a significantly increased 


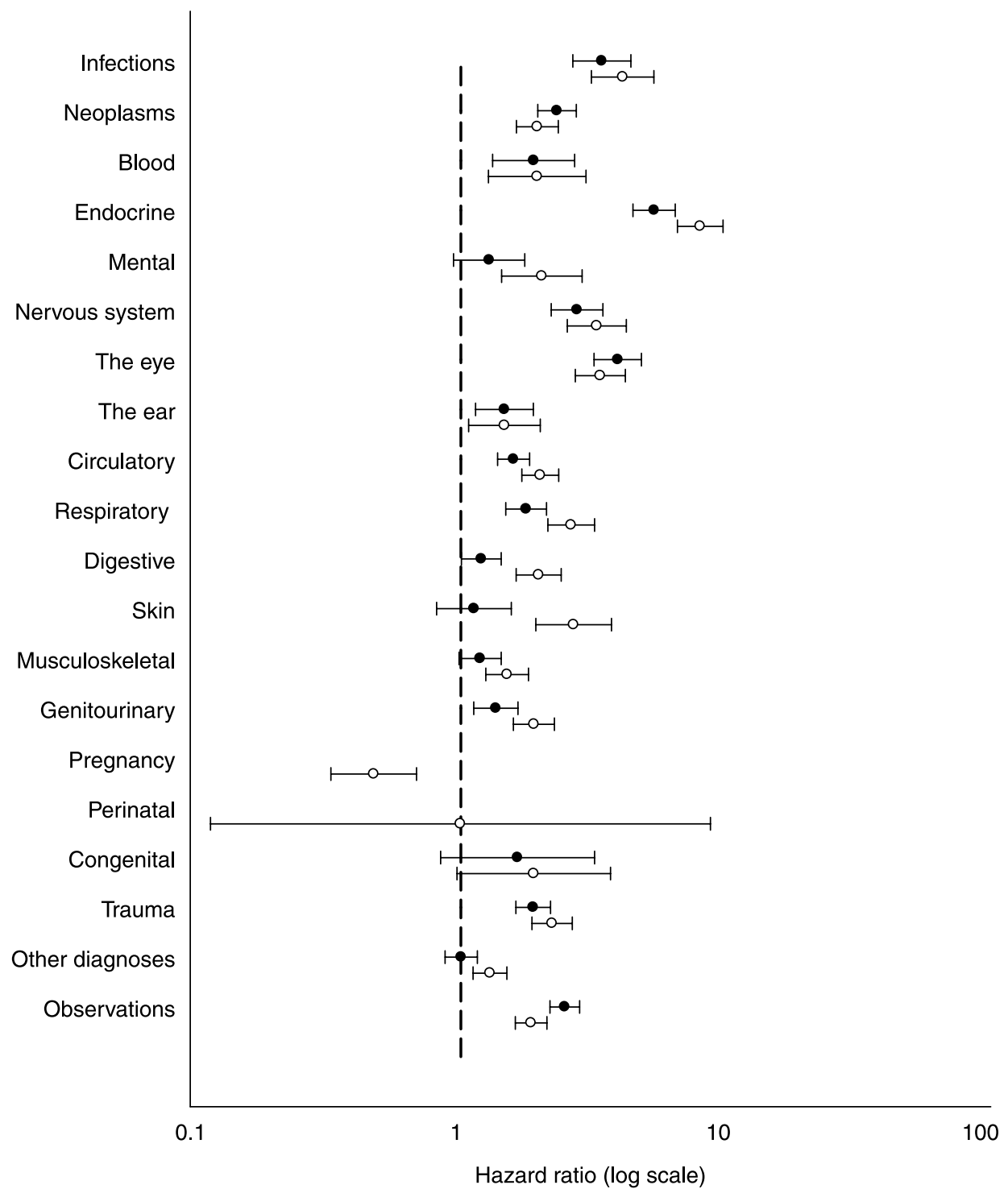

Figure 2 Hazard ratios of morbidity in adult onset (AO) GH deficiency (GHD) divided into 20 ICD-10 chapters. AO patients have an age at onset of GHD of 18 years or more. Males are represented in black circles and females in white circles.

morbidity in the thyroid among the patients. This may truly reflect a dysfunction of the thyroid gland, especially as the coding of pituitary dysfunction of the thyroid axis according to the ICD-10 was allocated in the sub-chapter of other endocrine organs, but we cannot exclude misclassification at the time of discharge. An increased number of cases with primary hypothyroidism after radiotherapy and chemotherapy for a childhood brain tumor have been described in a cohort of Danish patients (19). The increased morbidity caused by diabetes was not surprising, it being well established that patients with GHD have reduced insulin sensitivity (20), and this may contribute to the increased circulatory mortality in women (13), as may untreated hypogonadism (21). A recent Swedish study identified a significantly increased prevalence of diabetes in women $(n=351)$, but not in males $(n=399)$, possibly due to a smaller study size (22). Since a more manifest increase in morbidity due to diabetes, especially among males, could have been expected, the diagnosis of diabetes may be underdiagnosed in AO GHD males. Thus, a relatively increased circulatory morbidity and mortality in males compared with females would be expected. However, recently, we reported an increased mortality due to circulatory diseases in females in all age groups, whereas the circulatory mortality in males was increased only in the oldest age groups (12). Therefore, the relatively reduced diabetic morbidity in male patients compared with female patients may be correct. In line with the previously identified increased circulatory mortality, we also found significantly increased 
Table 3 Hazard ratios (HRs) of morbidity in both genders in childhood onset (CO) and adult onset (AO) growth hormone deficiency divided into 20 International Classification of Diseases-10 (ICD-10) chapters. Eventual changes in statistical significance of the HRs are shown using different approaches to the analysis.

\begin{tabular}{|c|c|c|c|c|c|c|c|c|}
\hline & \multicolumn{4}{|c|}{$\mathrm{co}$} & \multicolumn{4}{|c|}{ AO } \\
\hline & \multicolumn{2}{|c|}{ Males } & \multicolumn{2}{|c|}{ Females } & \multicolumn{2}{|c|}{ Males } & \multicolumn{2}{|c|}{ Females } \\
\hline & $\begin{array}{l}\text { First admission } \\
\text { patients/controls }\end{array}$ & $\mathrm{HR}$ & $\begin{array}{l}\text { First admission } \\
\text { patients/controls }\end{array}$ & $\mathrm{HR}$ & $\begin{array}{l}\text { First admission } \\
\text { patients/controls }\end{array}$ & $\mathrm{HR}$ & $\begin{array}{l}\text { First admission } \\
\text { patients/controls }\end{array}$ & $\mathrm{HR}$ \\
\hline Infections & $41 / 89$ & $2.2(1.5-3.2)^{a}$ & $29 / 69$ & $2.2(1.4-3.4)^{a}$ & $123 / 212$ & $3.5(2.7-4.5)$ & $106 / 156$ & $4.2(3.2-5.6)$ \\
\hline Cancer & $77 / 51$ & $9.0(6.1-13.3)$ & $53 / 51$ & $7.3(4.7-11.4)$ & $227 / 614$ & $2.3(2.0-2.8)$ & $170 / 582$ & $2.0(1.6-2.4)$ \\
\hline Blood diseases & $14 / 18$ & $3.6(1.8-7.4)^{b}$ & $3 / 17$ & $1.1(0.3-3.9)$ & $49 / 139$ & $1.9(1.3-2.7)^{\mathrm{a}}$ & $33 / 136$ & $2.0(1.3-3.1)^{a, c}$ \\
\hline Endocrine diseases & $106 / 31$ & $20.6(13.0-32.7)$ & $83 / 38$ & $21.0(12.6-35.0)$ & $273 / 366$ & $5.6(4.6-6.7)$ & $290 / 378$ & $8.4(6.9-10.3)$ \\
\hline Mental diseases & $24 / 45$ & $2.5(1.5-4.2)^{\mathrm{c}}$ & $20 / 30$ & $4.1(2.3-7.6)^{\mathrm{c}}$ & $56 / 237$ & $1.3(0.9-1.8)$ & $46 / 168$ & $2.1(1.4-2.9)$ \\
\hline Neurological diseases & $74 / 46$ & $10.4(6.8-15.8)$ & $53 / 37$ & $8.6(5.4-13.7)$ & $134 / 275$ & $2.8(2.2-3.5)$ & $104 / 208$ & $3.4(2.6-4.4)$ \\
\hline Diseases of the eye & $42 / 63$ & $3.1(2.0-4.6)^{\mathrm{b}}$ & $47 / 26$ & $13.2(7.5-23.4)$ & $192 / 321$ & $4.0(3.3-5.0)$ & $156 / 366$ & $3.5(2.8-4.3)$ \\
\hline Diseases of the ear & $36 / 40$ & $4.5(2.8-7.3)$ & $24 / 24$ & $5.6(3.0-10.2)$ & $90 / 388$ & $1.5(1.1-1.9)^{b}$ & $56 / 293$ & $1.5(1.1-2.0)^{a, b}$ \\
\hline Circulatory diseases & $34 / 49$ & $3.3(2.1-5.2)$ & $27 / 40$ & $5.2(3.0-9.1)$ & $309 / 1151$ & $1.6(1.4-1.8)^{a}$ & $241 / 851$ & $2.0(1.7-2.4)$ \\
\hline Pulmonary diseases & $56 / 146$ & $1.8(1.3-2.4)^{\mathrm{a}, \mathrm{c}}$ & $49 / 103$ & $2.6(1.8-3.7)$ & $193 / 616$ & $1.8(1.5-2.1)^{\mathrm{a}}$ & $154 / 412$ & $2.7(2.2-3.3)$ \\
\hline Gastrointestinal diseases & $61 / 161$ & $1.7(1.3-2.3)$ & $31 / 110$ & $1.5(1.0-2.3)$ & $181 / 777$ & $1.2(1.0-1.4)^{a, b, c, d, e}$ & $153 / 546$ & $2.0(1.6-2.4)$ \\
\hline Skin diseases & $23 / 73$ & $1.7(1.0-2.8)^{a, b, c, d}$ & $20 / 46$ & $2.4(1.4-4.2)^{c}$ & $48 / 205$ & $1.1(0.8-1.6)$ & $58 / 143$ & $2.7(2.0-3.8)$ \\
\hline Musculoskeletal diseases & $70 / 232$ & $1.5(1.1-1.9)^{\mathrm{c}}$ & $38 / 133$ & $1.5(1.0-2.2)^{b, c, d}$ & $158 / 688$ & $1.2(1.0-1.4)$ & $156 / 674$ & $1.5(1.3-1.8)^{a}$ \\
\hline Urogenital diseases & $35 / 92$ & $1.9(1.2-2.8)^{b}$ & $39 / 143$ & $1.7(1.2-2.4)^{\mathrm{a}, \mathrm{b}}$ & $155 / 596$ & $1.4(1.1-1.7)^{\mathrm{a}, \mathrm{b}}$ & $180 / 624$ & $1.9(1.6-2.3)$ \\
\hline Pregnancy and childbirth & $0 / 0$ & NA & $25 / 309$ & $0.3(0.2-0.5)$ & $0 / 0$ & NA & $34 / 259$ & $0.5(0.3-0.7)$ \\
\hline Diseases in the newborn & $4 / 0$ & $-^{f}$ & $2 / 2$ & $10.0(0.9-110.3)$ & $0 / 2$ & $-^{f}$ & $1 / 6$ & $1.0(0.1-9.2)$ \\
\hline Congenital malformations & $49 / 58$ & $4.8(3.2-7.2)$ & $36 / 19$ & $10.7(5.8-19.7)$ & $12 / 37$ & $1.7(0.8-3.3)$ & $12 / 36$ & $1.9(1.0-3.8)$ \\
\hline Trauma and intoxications & $89 / 162$ & $2.9(2.2-3.9)$ & $59 / 164$ & $2.2(1.6-3.0)$ & $274 / 882$ & $1.9(1.6-2.2)$ & $197 / 677$ & $2.3(1.9-2.7)$ \\
\hline Other diagnoses & $207 / 965$ & $1.1(1.0-1.3)$ & $101 / 474$ & $1.2(0.9-1.5)$ & $272 / 1332$ & $1.0(0.9-1.2)$ & $251 / 1179$ & $1.3(1.1-1.5)$ \\
\hline Observations & $189 / 521$ & $2.8(2.3-3.3)$ & $126 / 514$ & $1.6(1.3-2.0)$ & $432 / 1516$ & $2.5(2.2-2.9)$ & $340 / 1512$ & $1.9(1.6-2.2)$ \\
\hline
\end{tabular}

$\mathrm{CO}$ and $\mathrm{AO}$ are divided corresponding to an age at onset of $<18$ years and 18 years or more, respectively. Significant HRs are given in bold.

NA, not applicable.

Not significant when analyzing only admissions without any diagnoses used for the identification of patients.

Not significant after exclusion of patients with irradiation.

Not significant when analyzing only primary diagnoses.

Not significant after exclusion of patients with cancer diagnoses.

Not significant after exclusion of patients with acromegaly and Cushing's syndrome.

Due to few informative strata, statistical analysis was not possible. 
Table 4 Hazard ratios (HRs) of morbidity in both genders in adult onset growth hormone deficiency (GHD) divided into four age groups. Only overall significant morbidities are analyzed (Table 2). Adult onset (AO) is corresponding to an age at onset of 18 years or more of GHD. Significant HRs are given in bold.

\begin{tabular}{|c|c|c|c|c|c|c|c|c|}
\hline \multirow[b]{2}{*}{ ICD-10 chapters } & \multicolumn{4}{|c|}{ Males } & \multicolumn{4}{|c|}{ Females } \\
\hline & $<45$ years & $45-54$ years & 55-64 years & $65+$ years & $<45$ years & $45-54$ years & 55-64 years & $65+$ years \\
\hline Infections & $2.4(1.4-4.2)$ & $4.3(2.4-7.6)$ & $4.1(2.5-6.7)$ & $3.6(2.2-5.7)$ & $3.4(2.0-5.7)$ & $3.4(1.6-7.4)$ & $6.6(3.6-12.2)$ & $4.2(2.6-6.7)$ \\
\hline Cancer & $6.1(4.0-9.1)$ & $3.1(2.2-4.6)$ & $1.8(1.3-2.4)$ & $1.5(1.1-2.1)$ & $1.7(1.2-2.4)^{a}$ & $2.5(1.7-3.8)$ & $2.0(1.4-3.0)$ & $2.0(1.4-2.9)$ \\
\hline Blood diseases & $3.0(1.0-8.9)^{a}$ & $3.6(1.7-7.7)^{\mathrm{a}}$ & $1.2(0.6-2.6)$ & $1.6(0.9-2.9)$ & $2.1(0.7-6.0)$ & $1.8(0.5-5.6)$ & $3.9(1.6-9.3)$ & $1.5(0.8-3.0)$ \\
\hline Endocrine diseases & $13.1(8.6-20.0)$ & $6.3(4.3-9.2)$ & $4.7(3.2-6.9)$ & $2.9(2.1-4.2)$ & $17.7(11.4-27.5)$ & $10.1(6.2-16.5)$ & $8.8(5.6-13.9)$ & $4.4(3.1-6.1)$ \\
\hline Mental diseases & $-{ }^{\mathrm{b}}$ & $-\mathrm{b}$ & $-{ }^{\mathrm{b}}$ & $-^{\mathrm{b}}$ & $1.5(0.8-3.0)$ & $2.6(1.0-6.7)^{a}$ & $3.2(1.5-7.0)$ & $1.9(1.0-3.5)$ \\
\hline Neurological diseases & $6.3(4.1-9.7)$ & $3.4(2.1-5.5)$ & $1.5(0.9-2.3)$ & $1.5(0.9-2.5)$ & $4.5(2.8-7.1)$ & $4.5(2.5-8.1)$ & $2.2(1.3-3.9)$ & $2.7(1.6-4.6)$ \\
\hline Diseases of the eye & $7.4(4.6-11.7)$ & $4.3(2.7-6.9)$ & $3.6(2.4-5.3)$ & $2.8(1.9-4.2)$ & $5.3(3.2-8.5)$ & $6.2(3.5-10.8)$ & $3.9(2.5-6.1)$ & $2.0(1.4-2.9)$ \\
\hline Diseases of the ear & $2.7(1.5-5.0)$ & $1.8(1.0-3.1)^{a, c}$ & $1.4(0.9-2.2)$ & $1.0(0.6-1.6)$ & $2.4(1.2-4.9)^{a}$ & $2.2(1.1-4.3)^{\mathrm{C}}$ & $1.6(0.8-3.0)$ & $0.9(0.5-1.6)$ \\
\hline Circulatory diseases & $2.1(1.4-3.1)$ & $1.5(1.1-2.0)^{\mathrm{c}}$ & $1.3(1.0-1.7)$ & $1.8(1.4-2.2)$ & $2.9(2.1-4.2)$ & $1.7(1.2-2.6)^{\mathrm{C}}$ & $2.4(1.7-3.4)$ & $1.6(1.3-2.1)$ \\
\hline Pulmonary diseases & $3.1(1.9-4.8)$ & $2.5(1.7-3.9)$ & $1.5(1.1-2.1)^{\mathrm{a}}$ & $1.4(1.0-1.9)^{a}$ & $3.1(2.1-4.6)$ & $3.1(1.8-5.2)$ & $3.4(2.2-5.2)$ & $2.0(1.4-2.8)$ \\
\hline Gastrointestinal diseases & $1.8(1.3-2.6)$ & $0.9(0.6-1.4)$ & $1.2(0.9-1.7)$ & $1.0(0.7-1.4)$ & $3.3(2.3-4.8)$ & $1.7(1.0-2.7)^{\mathrm{c}}$ & $1.8(1.2-2.9)$ & $1.5(1.0-2.1)^{\mathrm{a}}$ \\
\hline Skin diseases & $-\mathrm{b}$ & $-\mathrm{b}$ & $-\mathrm{b}$ & $-\mathrm{b}$ & $2.4(1.4-4.0)$ & $3.2(1.4-7.8)$ & $2.6(1.2-5.6)^{\mathrm{a}}$ & $3.2(1.6-6.5)$ \\
\hline Musculoskeletal diseases & $-^{\mathrm{b}}$ & $-^{\mathrm{b}}$ & $-^{\mathrm{b}}$ & $-^{b}$ & $1.3(1.0-1.8)$ & $2.0(1.3-3.1)$ & $1.5(1.0-2.3)$ & $1.5(1.1-2.2)$ \\
\hline Urogenital diseases & $2.8(1.9-4.4)$ & $1.2(0.7-1.9)$ & $0.9(0.6-1.3)$ & $1.3(1.0-1.8)$ & $1.8(1.4-2.4)$ & $2.2(1.4-3.5)$ & $2.4(1.6-3.7)$ & $1.6(1.1-2.4)^{a}$ \\
\hline Pregnancy and childbirth & NA & NA & NA & NA & $0.4(0.3-0.7)$ & $-^{\mathrm{b}}$ & $-^{\mathrm{b}}$ & $-\mathrm{b}$ \\
\hline Trauma and intoxications & $3.1(2.2-4.4)$ & $1.7(1.2-2.4)$ & $1.6(1.2-2.1)$ & $1.9(1.4-2.5)$ & $2.5(1.8-3.5)$ & $3.6(0.8-16.2)$ & $1.0(0.1-9.2)$ & $1.2(0.1-10.7)$ \\
\hline Other diagnoses & & $-\mathrm{b}$ & $-\mathrm{b}$ & $-\mathrm{b}$ & $1.1(0.9-1.4)$ & $1.9(1.2-3.0)$ & $2.5(1.6-3.7)$ & $2.2(1.6-3.0)$ \\
\hline Observations & $2.9(2.3-3.6)$ & $2.4(1.8-3.1)$ & $2.4(1.8-3.2)$ & $2.4(1.8-3.2)$ & $1.6(1.3-1.9)$ & $1.5(1.1-2.2)$ & $1.4(1.0-2.0)^{\mathrm{a}}$ & $1.3(1.0-1.8)^{\mathrm{a}, \mathrm{c}}$ \\
\hline
\end{tabular}

NA, not applicable.

Not significant after exclusion of patients with cancer diagnoses.

'Not significant after exclusion of patients with acromegaly and Cushing's syndrome. 
Table 5 Selected sub-chapters describing morbidity in males and females with adult onset growth hormone deficiency compared with controls. Only sub-chapters with significant hazard ratios (HRs) are shown.

\begin{tabular}{|c|c|c|c|}
\hline \multirow[b]{2}{*}{ ICD-10 chapters } & \multicolumn{2}{|c|}{ HR } & \multirow[b]{2}{*}{ ICD-10 sub-chapters } \\
\hline & Males & Females & \\
\hline \multirow[t]{7}{*}{ Endocrine diseases } & $6.1(3.6-10.3)$ & $3.6(2.6-5.0)$ & Thyroid \\
\hline & $1.7(1.3-2.3)$ & $3.2(2.3-4.4)$ & Diabetes mellitus \\
\hline & & $3.9(1.5-10.5)$ & Other diseases in glucose regulation \\
\hline & $79.0(41.8-149.4)$ & $141.4(62.7-318.8)$ & Diseases in other endocrine organs \\
\hline & & $5.3(1.2-24.2)$ & Malnutrition \\
\hline & $4.6(2.3-9.3)$ & $2.4(1.4-4.2)$ & Obesity \\
\hline & $2.3(1.6-3.2)$ & $5.3(3.7-7.6)$ & Other metabolic diseases \\
\hline \multirow[t]{7}{*}{ Circulatory diseases } & $1.5(1.1-2.0)$ & $2.1(1.5-2.8)$ & Hypertension \\
\hline & $1.4(1.1-1.7)$ & $2.1(1.6-2.8)$ & Cardiac ischemia \\
\hline & $2.1(1.0-4.3)$ & & Pulmonary heart disease \\
\hline & $1.4(1.1-1.8)$ & $1.5(1.2-2.0)$ & Other causes of heart disease \\
\hline & $1.7(1.3-2.2)$ & $3.4(2.5-4.5)$ & Cerebrovascular diseases \\
\hline & & $2.4(1.7-3.6)$ & Diseases in arteries, arterioles, and capillaries \\
\hline & $1.5(1.1-2.1)$ & $1.8(1.2-2.5)$ & $\begin{array}{l}\text { Diseases in veins and lymph system not classified else- } \\
\text { where }\end{array}$ \\
\hline Pregnancy and childbirth & & $\begin{array}{l}0.4(0.2-0.8) \\
0.6(0.4-0.9)\end{array}$ & $\begin{array}{l}\text { Complicated delivery } \\
\text { Normal delivery }\end{array}$ \\
\hline
\end{tabular}

morbidity in five out of ten circulatory sub-chapters, including hypertension, cardiac ischemia, and cerebrovascular diseases, in both genders of AO patients. Furthermore, we identified a significantly increased risk of obesity in these patients, obesity also being an established clinical finding (23). Moreover, many hypopituitary patients become obese due to hypothalamic disorders, potential over-substitution with cortisol, or decreased physical activity due to decreased muscle mass and vitality. The increased morbidity caused by metabolic diseases (Table 5) is partly due to the fact that disturbances of the salt balance are included in this sub-chapter (data not shown). These findings are supported by an increased level of perceived health problems in hypopituitary patients (24).

Interestingly, there was a significantly decreased HR for all females with GHD in the chapter concerning pregnancy and childbirth. This may imply that they either have a reduced number of pregnancies or a reduced number of complications during pregnancies. Since in sub-chapters for AO patients a significantly decreased HR of normal, as well as complicated deliveries, had been identified, we conclude that these women have a decreased number of pregnancies. It remains to be clarified whether this was due to lack of interest, lack of partner, or reduced fertility. Previous studies have shown high-risk pregnancies in hypopituitary patients (25) and a reduced ovarian function in adult survivors of acute lymphoblastic leukemia in childhood (26). Reduced fertility in survivors of childhood cancer has been described earlier $(27,28)$, but data on fertility in adult women with hypopituitarism are scarce.

In females with AO GHD, we identified a significantly increased risk of fractures, while the risk of fractures in males was normal. It is well established that nonGH-treated male and female hypopituitary patients have a reduced BMD compared with controls (29-31), and that after 3 years of GH treatment of GHD patients BMD significantly increased in most re-examined regions (32). Furthermore, an increased risk of fractures among males and females with AO GHD not treated with GH compared with a sub-sample of the background population has been identified (33). However, in hypopituitary patients $60+$ years of age at examination, Toogood et al. found no alteration of BMD compared with controls (34). The increased risk of fractures in females but not in males may be due to a reduced percentage of properly treated females with hypogonadism ( $10 \%$ (males) vs $24 \%$ (females) of the adult hypogonadal patients younger than 55 years at entry were not treated with sex hormones; Table 1).

We identified HRs in selected groups of patients, either characterized by having isolated GHD, at any period having been operated, irradiated, or treated with GH. Here, it is important to emphasize that the timing of the treatment in question is not taken into consideration; thus, the patient might for instance have been irradiated at the end of the time at risk. In addition, the time period and dosage of GH are not included in this calculation. We would therefore not consider direct comparison between the groups feasible. However, it is noteworthy that the HRs seem to be severely increased in all the four subgroups in irradiated patients, and that GH treatment only seem to change the HR in AO females. However, the findings must be considered with caution.

Since all patients had been admitted to hospitals at least once, they had an increased risk of being diagnosed with other diseases. To control for this surveillance bias, we omitted all admissions related to the diagnoses used for the initial identification of the patients. We consider this a very conservative measure of morbidity. Furthermore, we analyzed primary diagnoses only; thus we focused on the most serious disease. The majority of significant HRs remained significant in both analyses. Furthermore, we 
Table 6 Total morbidity in selected groups of patients characterized by having isolated growth hormone (GH) deficiency, or at any period having been operated, or irradiated, or treated with GH. For comparison, total morbidity in all patients (Table 1) is included. All compared with controls.

\begin{tabular}{|c|c|c|c|c|}
\hline & \multicolumn{4}{|c|}{ HR } \\
\hline & \multicolumn{2}{|c|}{$\mathrm{CO}$} & \multicolumn{2}{|c|}{$\mathrm{AO}$} \\
\hline & Males & Females & Males & Females \\
\hline Isolated GHD & $2.5(1.9-3.3)$ & $2.4(1.7-3.4)$ & $3.9(2.6-6.0)$ & $2.7(1.8-4.1)$ \\
\hline Operated & $5.4(3.9-7.5)$ & $6.5(4.3-9.5)$ & $2.9(2.6-3.3)$ & $3.9(3.3-4.5)$ \\
\hline Irradiated & $6.0(4.3-8.3)$ & $6.8(4.3-10.8)$ & $5.1(3.9-6.6)$ & $5.2(3.9-7.0)$ \\
\hline $\mathrm{GH}$ treatment & $2.9(2.4-3.5)$ & $3.1(2.4-3.9)$ & $2.9(2.3-3.5)$ & $2.5(2.0-3.2)$ \\
\hline All patients & $3.1(2.7-3.7)$ & $3.2(2.6-3.9)$ & $2.9(2.6-3.2)$ & $3.2(2.8-3.6)$ \\
\hline
\end{tabular}

HR, hazard ratio.

identified hardly any change in total HR in the analyses including death as event. In conclusion, most HRs were significant even using the most conservative analyses. However, surveillance bias may still be present, and therefore the interpretation of specific HRs including CIs should be undertaken carefully. The diagnostic criteria for the definition of GHD changed over time, and the GHD patients were identified using an algorithm as described previously (12). The validity of the registers is generally high but caution should be taken in interpretation of data. Physicians may have an increased clinical awareness of certain clinical consequences in the GHD patients, for instance diabetes mellitus. The size of this surveillance bias is difficult to estimate and may overestimate morbidity in the GHD patients. Every registration increases the chance of additional diagnoses, diagnoses which in themselves would not result in an admission. Even significantly increased HRs may be subject to this phenomenon, especially when registrations are limited. Patients with known diseases have per se a higher risk of being diagnosed with other diseases (18). For the definition of morbidity, we used lag time until first relevant diagnosis at a hospital. The number of diagnoses or the impact of diagnosis could have been applied as well. The number of diagnoses is increased in the GHD patients, and the impact of diagnoses is difficult to estimate; thus, we did not perform these analyses. It is important to emphasize that patients are defined as such from the date of entry, an arbitrary date applied to ensure identical epidemiological terms for all patients, and not from the date of onset of GHD. Thus, only persons with GHD, who survive until the date of entry, are included in the analyses. This may to some extent underestimate morbidity.

The strength of the present study is the national identification of GHD patients applying the same approach to all Danish hospitals. In Denmark, the access to hospitals is free and all citizens are covered without expenses. Only a very limited number of admissions that increased to 1\% in 2003 were at private hospitals (source: www.sst.dk). Possible socio-economic differences between patients and controls can therefore be neglected regarding choice of hospital. All controls are randomly identified from all Danish citizens, and the matching for age and gender of patients and controls results in identical conditions, including calendar time. Using such an approach, the annual number of persons lost to follow-up is limited, and none in this study was lost. The use of central person number uniquely links patients as well as controls to information in NPR. NPR covered ambulatory patients only from 1995 onwards; thus an increase in registrations after 1995 is expected. Similarly, the ICD changed from ICD-8 to ICD-10. Both of these conditions, as well as the quality of data, are considered identical for patients and controls. However, we believe we have taken all possible precautions when analyzing data.

In conclusion, this nationwide study identified a significantly increased morbidity in males and females, with childhood or AO GHD. For all the four subgroups, morbidity was significantly increased in infectious, cancer, endocrine, neurological, eye, ear, circulatory, pulmonary and urogenital diseases, trauma, and observations. Within the patients, females with AO GHD had a significantly increased morbidity compared with males. There was a significantly increased risk of fractures in females with AO GHD, but not in males. We consider the overall increased morbidity in the GHD patients as definitive. It remains to be clarified whether the increased morbidity can be modified by GH supplementation or optimized substitution of other axes.

\section{Acknowledgements}

All departments with patients registered are gratefully thanked for their positive attitude towards the project. Kirstine Stochholm was supported by an unrestricted research grant from Novo Nordisk A/S, Denmark.

\section{References}

1 de Boer H, Blok GJ, van Lingen A, Teule GJ, Lips P \& van der Veen EA. Consequences of childhood-onset growth hormone deficiency for adult bone mass. Journal of Bone and Mineral Research 19949 1319-1326. 
2 Hyer SL, Rodin DA, Tobias JH, Leiper A \& Nussey SS. Growth hormone deficiency during puberty reduces adult bone mineral density. Archives of Disease in Childhood $1992671472-1474$.

3 Murray RD, Brennan BM, Rahim A \& Shalet SM. Survivors of childhood cancer: long-term endocrine and metabolic problems dwarf the growth disturbance. Acta Paediatrica 199988 5-12.

4 Kendall-Taylor P, Jonsson PJ, Abs R, Erfurth EM, KoltowskaHaggstrom M, Price DA \& Verhelst J. The clinical, metabolic and endocrine features and the quality of life in adults with childhoodonset craniopharyngioma compared with adult-onset craniopharyngioma. European Journal of Endocrinology 2005152 557-567.

5 Jorgensen JO, Pedersen SA, Thuesen L, Jorgensen J, IngemannHansen T, Skakkebaek NE \& Christiansen JS. Beneficial effects of growth hormone treatment in GH-deficient adults. Lancet 19891 1221-1225.

6 Salomon F, Cuneo RC, Hesp R \& Sonksen PH. The effects of treatment with recombinant human growth-hormone on bodycomposition and metabolism in adults with growth-hormone deficiency. New England Journal of Medicine 1989321 1797-1803.

7 Colao A, Di Somma C, Salerno M, Spinelli L, Orio F \& Lombardi G. The cardiovascular risk of GH-deficient adolescents. Journal of Clinical Endocrinology and Metabolism 200287 3650-3655.

8 Johannsson G, Rosen T \& Bengtsson BA. Individualized dose titration of growth hormone $(\mathrm{GH})$ during $\mathrm{GH}$ replacement in hypopituitary adults. Clinical Endocrinology 199747 571-581.

9 Lucidi P, Lauteri M, Laureti S, Celleno R, Santoni S, Volpi E, Angeletti G, Santeusanio F \& De Feo P. A dose-response study of growth hormone $(\mathrm{GH})$ replacement on whole body protein and lipid kinetics in GH-deficient adults. Journal of Clinical Endocrinology and Metabolism $1998 \mathbf{8 3} 353-357$.

10 Svensson J, Bengtsson BA, Rosen T, Oden A \& Johannsson G. Malignant disease and cardiovascular morbidity in hypopituitary adults with or without growth hormone replacement therapy. Journal of Clinical Endocrinology and Metabolism 2004 89 3306-3312.

11 Swerdlow AJ, Higgins CD, Adlard P \& Preece MA. Risk of cancer in patients treated with human pituitary growth hormone in the UK, 1959-85: a cohort study. Lancet 2002360 273-277.

12 Stochholm K, Gravholt CH, Laursen T, Jorgensen JO, Laurberg P, Andersen M, Kristensen LØ, Feldt-Rasmussen U, Christiansen JS, Frydenberg M \& Green A. Incidence of GH Deficiency - a Nationwide Study. European Journal of Endocrinology 2006155 61-71.

13 Stochholm K, Gravholt CH, Laursen T, Laurberg P, Andersen M, Kristensen L, Feldt-Rasmussen U, Christiansen JS, Frydenberg M \& Green A. Mortality in growth hormone deficiency - a nationwide study. European Journal of Endocrinology 2007 157 9-18.

14 Sonksen $\mathrm{PH} \&$ Christiansen JS. Consensus guidelines for the diagnosis and treatment of adults with growth hormone deficiency. Growth Hormone Research Society. Growth Hormone and IGF Research 19988 (Suppl B) 89-92.

15 Society GR. Consensus Guidelines for the Diagnosis and Treatment of Growth Hormone (GH) Deficiency in Childhood and Adolescence: Summary Statement of the GH Research Society. Journal of Clinical Endocrinology and Metabolism 200085 3990-3993.

16 Littley MD, Shalet SM, Beardwell CG, Ahmed SR, Applegate G \& Sutton ML. Hypopituitarism following external radiotherapy for pituitary tumours in adults. Quarterly Journal of Medicine 198970 145-160.

17 Hartman ML, Crowe BJ, Biller BM, Ho KK, Clemmons DR \& Chipman JJ. Which patients do not require a $\mathrm{GH}$ stimulation test for the diagnosis of adult GH deficiency? Journal of Clinical Endocrinology and Metabolism $2002 \mathbf{8 7} 477-485$.

18 Berkson J. Limitations of the application of fourfold table analysis to hospital data. Biometrics Bulletin 19492 47-53.

19 Schmiegelow M, Feldt-Rasmussen U, Rasmussen AK, Poulsen HS \& Muller J. A population-based study of thyroid function after radiotherapy and chemotherapy for a childhood brain tumor. Journal of Clinical Endocrinology and Metabolism 200388 136140.

20 Beshyah SA, Henderson A, Niththyananthan R, Skinner E, Anyaoku V, Richmond W, Sharp P \& Johnston DG. The effects of short and long-term growth hormone replacement therapy in hypopituitary adults on lipid metabolism and carbohydrate tolerance. Journal of Clinical Endocrinology and Metabolism 1995 $80356-363$.

21 Rosen T \& Bengtsson BA. Premature mortality due to cardiovascular disease in hypopituitarism. Lancet 1990336 285-288.

22 Holmer H, Svensson J, Rylander L, Johannsson G, Rosen T, Bengtsson BA, Thoren M, Hoybye C, Degerblad M, Bramnert M, Hagg E, Engstrom BE, Ekman B, Norrving B, Hagmar L \& Erfurth EM. Non-fatal stroke, cardiac disease, and diabetes mellitus in hypopituitary patients on hormone replacement including growth hormone. Journal of Clinical Endocrinology and Metabolism 200792 3560-3567.

23 Beshyah SA, Freemantle C, Thomas E, Rutherford O, Page B, Murphy M \& Johnston DG. Abnormal body composition and reduced bone mass in growth hormone deficient hypopituitary adults. Clinical Endocrinology 199542 179-189.

24 Rosen T, Wiren L, Wilhelmsen L, Wiklund I \& Bengtsson BA. Decreased psychological well-being in adult patients with growth hormone deficiency. Clinical Endocrinology $199440111-116$.

25 Overton CE, Davis CJ, West C, Davies MC \& Conway GS. High risk pregnancies in hypopituitary women. Human Reproduction 2002 17 1464-1467.

26 Wallace WH, Shalet SM, Tetlow LJ \& Morris-Jones PH. Ovarian function following the treatment of childhood acute lymphoblastic leukaemia. Medical and Pediatric Oncology 199321 333-339.

27 Byrne J, Mulvihill JJ, Myers MH, Connelly RR, Naughton MD, Krauss MR, Steinhorn SC, Hassinger DD, Austin DF \& Bragg K. Effects of treatment on fertility in long-term survivors of childhood or adolescent cancer. New England Journal of Medicine 1987317 1315-1321.

28 Stevens MCG, Mahler H \& Parkes S. The health status of adult survivors of cancer in childhood. European Journal of Cancer 1998 34 694-698.

29 Bing-You RG, Denis MC \& Rosen CJ. Low bone mineral density in adults with previous hypothalamic-pituitary tumors: correlations with serum growth hormone responses to GH-releasing hormone, insulin-like growth factor I, and IGF binding protein 3. Calcified Tissue International $1993 \mathbf{5 2} 183-187$.

30 Holmes SJ, Economou G, Whitehouse RW, Adams JE \& Shalet SM. Reduced bone mineral density in patients with adult onset growth hormone deficiency. Journal of Clinical Endocrinology and Metabolism 199478 669-674.

31 Beshyah SA, Freemantle C, Thomas E, Rutherford O, Page B, Murphy M \& Johnston DG. Abnormal body composition and reduced bone mass in growth hormone deficient hypopituitary adults. Clinical Endocrinology 199542 179-189.

32 Rahim A, Holmes SJ, Adams JE \& Shalet SM. Long-term change in the bone mineral density of adults with adult onset growth hormone $(\mathrm{GH})$ deficiency in response to short or long-term $\mathrm{GH}$ replacement therapy. Clinical Endocrinology $1998 \mathbf{4 8} 463-469$.

33 Rosen T, Wilhelmsen L, Landin-Wilhelmsen K, Lappas G \& Bengtsson BA. Increased fracture frequency in adult patients with hypopituitarism and GH deficiency. European Journal of Endocrinology $1997137240-245$.

34 Toogood AA, Adams JE, O'Neill PA \& Shalet SM. Elderly patients with adult-onset growth hormone deficiency are not osteopenic. Journal of Clinical Endocrinology and Metabolism $1997821462-1466$.

Received 11 November 2007

Accepted 17 December 2007 